\title{
Finite-Time Second-Order Sliding Mode Controllers for Spacecraft Attitude Tracking
}

\author{
Chutiphon Pukdeboon \\ Department of Mathematics, Faculty of Applied Sciences, King Mongkut's University of Technology North Bangkok, \\ Bangkok 10800, Thailand \\ Correspondence should be addressed to Chutiphon Pukdeboon; cpd@kmutnb.ac.th
}

Received 9 April 2013; Accepted 20 June 2013

Academic Editor: Yuqiang Wu

Copyright ( $(2013$ Chutiphon Pukdeboon. This is an open access article distributed under the Creative Commons Attribution License, which permits unrestricted use, distribution, and reproduction in any medium, provided the original work is properly cited.

\begin{abstract}
The attitude tracking control problem of a spacecraft nonlinear system with external disturbances and inertia uncertainties is studied. Two robust attitude tracking controllers based on finite-time second-order sliding mode control schemes are proposed to solve this problem. For the first controller, smooth super twisting control is applied to quaternion-based spacecraft-attitude-tracking maneuvers. The second controller is developed by adding linear correction terms to the first super twisting control algorithm in order to improve the dynamic performance of the closed-loop system. Both controllers are continuous and, therefore, chattering free. The concepts of a strong Lyapunov function are employed to ensure a finite-time convergence property of the proposed controllers. Theoretical analysis shows that the resulting control laws have strong robustness and disturbance attenuation ability. Numerical simulations are also given to demonstrate the performance of the proposed control laws.
\end{abstract}

\section{Introduction}

In recent years, attitude tracking control of a rigid spacecraft has attached a great deal of attention. To improve the performance of closed-loop systems, various nonlinear control approaches have been proposed (see, e.g., [1-5]). The PD control approach was used in [6] to achieve asymptotic stability in the presence of model uncertainties, while passivity-based control was presented in [7] to guarantee the asymptotical convergence of attitude tracking without angular velocity measurements. In [8], a nonlinear controller was proposed for large-angle attitude control of a spacecraft. The nonlinear control parameters were determined using the linear matrix inequality (LMI) approach. Later, Luo et al. [9] developed an $H_{\infty}$ inverse optimal adaptive controller for attitude tracking of spacecraft. Adaptive control and nonlinear $H_{\infty}$ control were merged to design robust optimal controllers. These controllers can achieve asymptotic attitude tracking with an uncertain inertia matrix and can ensure boundedness. In [10], the attitude tracking and disturbance rejection problems of spacecraft were investigated. An adaptive controller was developed for the stabilization problem providing asymptotic rejection for a class of external disturbances by designing a compensator. The attitude tracking problem without angular velocity measurements was studied (see, e.g., $[11,12])$. In [11], the authors developed an adaptive control law which incorporated a velocity filter from attitude measurements to ensure the asymptotic convergence of the attitude and angular velocity tracking errors. In [12], velocity filters were designed to compensate for the unmeasurable angular velocity and the proposed control law provided a semiglobal stability result. In [13], integrator backstepping-based control design was developed for application to the attitude maneuver problem.

In practical situations, the inertia matrix of the spacecraft cannot be exactly known, and the spacecraft is always subject to external disturbances. Sliding mode control (SMC) has been shown to be an effective method for the control of uncertain dynamical systems. Its ability to reject disturbances and parameter variations is useful for practical applications. Based on SMC schemes, the controller designs of attitude tracking control were proposed in $[14,15]$. These control laws are asymptotically stable which means that the rotational motion of a rigid body can be driven to track a given desired trajectory as time trends to infinity. Obviously, it is more 
desirable to use finite-time control schemes, since these techniques ensure that tracking is achieved in finite time.

A recently developed technique for finite-time stabilization is terminal sliding mode (TSM) control $[16,17]$ which can guarantee that the system states converge to the equilibrium point in finite-time. In [18-20], controllers were designed using the TSM approach and a quaternion representation to achieve finite time convergence of both attitude and angular velocity tracking errors. However, SMC induces control chattering due to the discontinuous control law. In mechanical systems, such control signals are undesirable because they can cause damage and accelerate wear. The SMC theory has been extended in recent years to incorporate a new technique which is known as higher-order sliding mode control (HOSMC) [21, 22]. This technique preserves the main advantages of SMC and also yields improved accuracy and performance. Various real-life applications have been controlled in a practical implementation of HOSMC (see, e.g., [23-25]). However, higher-order sliding mode controllers (HOSMC) of nonlinear spacecraft systems have been rarely studied. Quasicontinuous second- and third-order sliding controllers have been presented in [26] to solve the attitude tracking control problem.

In this paper, we study spacecraft attitude tracking controller designs using HOSMC. Two finite-time second-order sliding mode control (SOSMC) algorithms are developed to design attitude tracking controllers of a rigid spacecraft. For the first controller, the smooth super twisting (ST) algorithm is applied to deal with quaternion-based spacecraft-attitudetracking maneuvers. This control approach was proposed in [27] and the weak Lyapunov function was used to prove the stability. It is well known that a weak Lyapunov function ensures stability but cannot guarantee asymptotic stability, and finite-time convergence. The LaSalle theorem is needed to guarantee asymptotic stability and homogeneity properties are required to prove the finite-time convergence [28]. In this research, a strong Lyapunov design [29] is proposed which ensures the finite-time stability of this control law without the need for the LaSalle theorem and homogeneity properties, and even in the case when the bounded external perturbations are taken into account. In addition, a smooth modified super twisting (MST) sliding mode scheme is developed by adding linear correction terms to the first super twisting control algorithm to improve the dynamic performance of the closed-loop system. Hence, using strong Lyapunov designs, both proposed controllers can guarantee finite-time convergence to a given desired attitude motion of a rigid spacecraft.

This paper is organized as follows. Section 2 contains a description of the dynamic equations and the kinematics of attitude for the spacecraft attitude tracking problem ([30, 31]). Section 3 states the control problem. A finite-time attitude tracking controller is designed using the smooth ST algorithm. The finite convergence of the proposed controller for the resulting closed-loop system is proved theoretically. In Section 4, a controller design based on the smooth MST algorithm is provided. The stability of the closed-loop system is analyzed. Numerical simulations are given in Section 5 to demonstrate the performance of the proposed controllers. In Section 6, we present conclusions.

\section{Mathematical Model of Spacecraft Attitude Tracking Control}

2.1. Kinematics of the Attitude Error. We now briefly explain the use of quaternions for description of the attitude error. We define the quaternion $Q=\left[\begin{array}{ll}q^{T} & q_{4}\end{array}\right]^{T} \in \mathscr{R}^{3} \times \mathscr{R}$ with $q=\left[\begin{array}{lll}q_{1} & q_{2} & q_{3}\end{array}\right]^{T} \in \mathscr{R}^{3}$ and

$$
Q_{r}=\left[\begin{array}{ll}
q_{r}^{T} & q_{4 r}
\end{array}\right]^{T}
$$

where $q_{r}=\left[\begin{array}{lll}q_{1 r} & q_{2 r} & q_{3 r}\end{array}\right]^{T} \in \mathscr{R}^{3}$ is the desired reference attitude. The quaternion for the attitude error is $Q_{e}=$ $\left[\begin{array}{ll}q_{e}^{T} & q_{4 e}\end{array}\right]^{T} \in \mathscr{R}^{3} \times \mathscr{R}$ with $q_{e}=\left[\begin{array}{lll}q_{1 e} & q_{2 e} & q_{3 e}\end{array}\right]^{T} \in \mathscr{R}^{3}$. Using the multiplication law for quaternions, we then obtain [32]

$$
Q_{e}=\left[\begin{array}{c}
q_{4 r} q-q_{4} q_{r}-q_{r}^{\times} q \\
q_{4} q_{4 r}+q^{T} q_{r}
\end{array}\right]
$$

subject to the constraint

$$
Q_{e}^{T} Q_{e}=\left(q^{T} q+q_{4}^{2}\right)\left(q_{r}^{T} q_{r}+q_{4 r}^{2}\right)=1
$$

Note that a quaternion consists of the scalar $q_{4}$ and the threedimensional vector $q$, so it has four components. The scalar term is used for avoidance of singular points in the attitude representation [33]. The quaternion kinematics equation is required to be solved for all four components. However, to indicate the maneuver of the spacecraft, it is sufficient to use only the vector $q$ because this vector properly represents both Euler axis and Euler angle. Furthermore, the scalar $q_{4}$ can be calculated easily using the vector $q$ and the condition $\|Q\|=1$. For more details of quaternion and other attitude representation, see [31, 32].

The kinematic equation for the attitude error can then be expressed as (see, e.g., $[10,31])$

$$
\dot{Q}_{e}=\frac{1}{2}\left[\begin{array}{c}
T\left(Q_{e}\right) \\
-q_{e}^{T}
\end{array}\right] \omega_{e},
$$

where $I_{3}$ is the $3 \times 3$ identity matrix and $T\left(Q_{e}\right)=q_{e}^{\times}+q_{4 e} I_{3}$.

To avoid the singularity of $T\left(Q_{e}\right)$ that will occur at $q_{4 e}=$ 0 , we let the attitude of the spacecraft be restricted to the workspace $W$ defined by [23]

$$
\begin{gathered}
W=\left\{Q_{e} \mid Q_{e}=\left[\begin{array}{ll}
q_{e}^{T} & q_{4 e}
\end{array}\right]^{T},\left\|q_{e}\right\| \leq \beta<1,\right. \\
\left.q_{4 e} \geq \sqrt{1-\beta^{2}}>0\right\}
\end{gathered}
$$

where $\beta$ is a positive constant.

2.2. Dynamic Equations of the Error Rate. In [31], the dynamic equation for a rigid spacecraft rotating under the influence of body-fixed devices is given as

$$
J \dot{\omega}=-\omega^{\times} J \omega+u+d,
$$

where $\omega=\left[\begin{array}{lll}\omega_{1} & \omega_{2} & \omega_{3}\end{array}\right]^{T}$ is the angular rate of the spacecraft, $u=\left[\begin{array}{lll}u_{1} & u_{2} & u_{3}\end{array}\right]^{T}$ represents the control vector, 
$d=\left[\begin{array}{lll}d_{1} & d_{2} & d_{3}\end{array}\right]^{T}$ are bounded disturbances, $J$ is the inertia matrix, and the skew-symmetric matrix $\omega^{\times}$is defined by

$$
\omega^{\times}=\left[\begin{array}{ccc}
0 & -\omega_{3} & \omega_{2} \\
\omega_{3} & 0 & -\omega_{1} \\
-\omega_{2} & \omega_{1} & 0
\end{array}\right] .
$$

We denote $\omega_{r}=\left[\begin{array}{lll}\omega_{1 r} & \omega_{2 r} & \omega_{3 r}\end{array}\right]^{T}$ as the desired reference rate and $\omega_{e}=\omega-C \omega_{r}$ with $C=\left(q_{4 e}^{2}-2 q_{e}^{T} q_{e}\right) I_{3}+2 q_{e} q_{e}^{T}-$ $2 q_{4 e} q_{e}^{\times}$as the error rate. Then, substitution into (6) gives the dynamic equation of the error rate in the form [32]

$$
\begin{aligned}
J \dot{\omega}_{e}= & -\left(\omega_{e}+C \omega_{r}\right)^{\times} J\left(\omega_{e}+C \omega_{r}\right)+u+d \\
& +J\left(\omega_{e}^{\times} C \omega_{r}-C \dot{\omega}_{r}\right) .
\end{aligned}
$$

Then, taking the following coordinate transformation suggested in [10]

$$
x=\omega_{e}+\lambda q_{e}
$$

gives

$$
\begin{gathered}
\dot{q}_{e}=\frac{1}{2}\left(q_{4 e} I_{3}+q_{e}^{\times}\right) \omega_{e}, \quad \dot{q}_{4 e}=-\frac{1}{2} q_{e}^{T} \omega_{e}, \\
J \dot{x}=-\left(\omega_{e}+C \omega_{r}\right)^{\times} J\left(\omega_{e}+C \omega_{r}\right)+J\left(\omega_{e}^{\times} C \omega_{r}-C \dot{\omega}_{r}\right) \\
\times \frac{1}{2} \lambda J\left(q_{4 e} I_{3}+q_{e}^{\times}\right) \omega_{e}+u+d,
\end{gathered}
$$

where $\lambda$ is a positive constant.

Next, consider the inertia matrix containing the parameter uncertainty in the form $J=J_{0}+\Delta J$, where $J_{0}$ is the known constant matrix which is selected nonsingular, and $\Delta J$ denotes the unmatched uncertainty. Thus, the dynamic equation (11) can be written as

$$
\begin{aligned}
\left(J_{0}+\Delta J\right) \dot{x}= & -\left(\omega_{e}+C \omega_{r}\right)^{\times}\left(J_{0}+\Delta J\right)\left(\omega_{e}+C \omega_{r}\right) \\
& +\left(J_{0}+\Delta J\right)\left(\omega_{e}^{\times} C \omega_{r}-C \dot{\omega}_{r}\right) \\
& \times \frac{\lambda}{2}\left(J_{0}+\Delta J\right)\left(q_{4 e} I_{3}+q_{e}^{\times}\right) \omega_{e}+u+d .
\end{aligned}
$$

Note that $\left(J_{0}+\Delta J\right)^{-1}$ can be expressed as

$$
\left(J_{0}+\Delta J\right)^{-1}=J_{0}^{-1}+\Delta \tilde{J},
$$

where $\Delta \widetilde{J}$ is also an uncertainty that can be found by expanding the first term of the expression below:

$$
\begin{aligned}
\Delta \widetilde{J} & =\left(J_{0}+\Delta J\right)^{-1}-J_{0}^{-1} \\
& =J_{0}^{-1}\left(I_{3}+J_{0}^{-1} \Delta J\right)^{-1}-J_{0}^{-1}
\end{aligned}
$$

leading to

$$
\Delta \widetilde{J}=\left[\left(I_{3}+J_{0}^{-1} \Delta J\right)^{-1}-I_{3}\right] J_{0}^{-1}
$$

provided that $\|\Delta J\|<1 /\left\|J_{0}^{-1}\right\|$. This is a sufficient condition for the existence of $\Delta \widetilde{J}$. From Banach perturbation lemma, one can obtain $\left\|\left(I_{3}+J_{0}^{-1} \Delta J\right)^{-1}\right\| \leq 1 /\left(1-\left\|J_{0}^{-1}\right\|\|\Delta J\|\right)$.

Thus, with some simple algebraic manipulations to (12), one obtains [19]

$$
\dot{x}=F+B+J_{0}^{-1} u+\bar{d}
$$

where

$$
\begin{gathered}
F=J_{0}^{-1}\left[-\left(\omega_{e}+C \omega_{r}\right)^{\times} J_{0}\left(\omega_{e}+C \omega_{r}\right)\right. \\
+J_{0}\left(\omega_{e}^{\times} C \omega_{r}-C \dot{\omega}_{r}\right) \\
\left.+\frac{1}{2} \lambda J_{0}\left(q_{4 e} I_{3}+q_{e}^{\times}\right) \omega_{e}\right], \\
B=J_{0}^{-1}\left[-\left(\omega_{e}+C \omega_{r}\right)^{\times} \Delta J\left(\omega_{e}+C \omega_{r}\right)\right. \\
\left.+\Delta J\left(\omega_{e}^{\times} C \omega_{r}-C \dot{\omega}_{r}\right)+\frac{1}{2} \lambda \Delta J\left(q_{4 e} I_{3}+q_{e}^{\times}\right) \omega_{e}\right] \\
+\Delta \widetilde{J}\left[-\left(\omega_{e}+C \omega_{r}\right)^{\times} J\left(\omega_{e}+C \omega_{r}\right)\right. \\
\left.+J\left(\omega_{e}^{\times} C \omega_{r}-C \dot{\omega}_{r}\right)+\frac{1}{2} \lambda J\left(q_{4 e} I_{3}+q_{e}^{\times}\right) \omega_{e}\right] \\
+\Delta \widetilde{J} u, \quad \bar{d}=J_{0}^{-1} d+\Delta \widetilde{J} d .
\end{gathered}
$$

The system (16) includes both parameter uncertainty $B$ and external disturbance $\bar{d}$. In this section, the uncertainty and disturbance are lumped together as the total disturbances $\widetilde{d}$, and thus the simplified system is obtained:

$$
\dot{x}=F+B_{0} u+\tilde{d},
$$

where $\widetilde{d}=B+\bar{d}$ and $B_{0}=J_{0}^{-1}$.

2.3. Finite-Time Stability. We now restate the concepts related to finite-time stability $[30,34]$.

Definition 1 (see [30]). Consider a time invariant system in the form of

$$
\dot{x}=f(x), \quad f(0)=0, \quad x \in \mathscr{R}^{n},
$$

where $f: D \rightarrow \mathscr{R}^{n}$ is continuous on an open neighborhood $D$ of the origin. The equilibrium $x=0$ of the system is (locally) finite-time stable if (i) it is asymptotically stable, in $\widehat{U}$, an open neighborhood of the origin, with $\widehat{U} \subseteq D$; (ii) it is finite-time convergent in $\widehat{U}$; that is, for any initial condition $x_{0} \in \widehat{U} \backslash\{0\}$, there is a settling time $T>0$ such that every solution $x\left(t, x_{0}\right)$ of system (19) is defined with $x\left(t, x_{0}\right) \in$ $\widehat{U} \backslash\{0\}$ for $t \in[0, T)$ and satisfies

$$
\lim _{t \rightarrow T\left(x_{0}\right)} x\left(t, x_{0}\right)=0
$$


and $x\left(t, x_{0}\right)=0$, if $t \geq T$. Moreover, if $\widehat{U}=\mathscr{R}^{n}$, the origin is globally finite-time stable.

Definition 2. Consider a controlled system

$$
\dot{x}=f(x)+g(x) u, \quad x \in \mathscr{R}^{n}, u \in \mathscr{R}^{m}
$$

with $g(x) \neq 0$. It is finite-time stabilizable if there is a feedback law $u(x)$ such that $x=0$ is a (locally) finite-time stable equilibrium of closed-loop system.

Lemma 3 (see [34]). Consider the nonlinear system described in (19). Suppose that there is a $C^{1}$ function $V(x): D \rightarrow \mathscr{R}$ defined on a neighborhood $\widehat{U} \subseteq D$ of the origin such that the following conditions hold: (i) $V(x)$ is positive definite on $D \subseteq$ $\mathscr{R}^{n}$; (ii) there are real numbers $\varrho>0$ and $0<\iota<1$, such that

$$
\dot{V}+\varrho V^{l} \leq 0, \quad x \in \widehat{U} \backslash\{0\} .
$$

Then, the origin of system (19) is finite-time stable. If $D=\mathscr{R}^{n}$ and $V(x)$ is radially unbounded, then the origin of system (19) is globally finite-time stable.

\section{Smooth ST Algorithm}

In this section, the problem of attitude tracking control for a rigid spacecraft is studied. The control objective is to design the control input $u_{f}$ so that the trajectory tracking errors of the state with respect to the desired state will converge to a desired region in finite time. A proof of finite convergence of the closed-loop system is provided based on the strong Lyapunov function.

In this section, we develop an attitude tracking control based on the ST algorithm. The goal is to enforce the sliding mode on the manifold

$$
s=x
$$

where $s=\left[\begin{array}{lll}s_{1} & s_{2} & s_{3}\end{array}\right]^{T} \in \mathscr{R}^{3}$.

We now ignore the disturbance term in (18). Letting $u=$ $u_{f}$, the proposed controller is given by the following:

$$
\begin{aligned}
u_{f}=\left(B_{0}\right)^{-1}( & -F-K_{1} \operatorname{sign}(s)^{(p-1) / p} \\
& \left.-K_{2} \int_{0}^{t} \operatorname{sign}(s(\tau))^{(p-2) / p} d \tau\right),
\end{aligned}
$$

where $p \geq 2$. The function $\operatorname{sign}(s)^{r}$ is defined as

$$
\begin{aligned}
& \operatorname{sign}(s)^{r} \\
& \quad=\left[\left|s_{1}\right|^{r} \operatorname{sign}\left(s_{1}\right)\left|s_{2}\right|^{r} \operatorname{sign}\left(s_{2}\right), \ldots,\left|s_{m}\right|^{r} \operatorname{sign}\left(s_{m}\right)\right]^{T}
\end{aligned}
$$

with $0<r<1$. The gain matrices $K_{1}=\operatorname{diag}\left(k_{11}, k_{12}, k_{13}\right)$ and $K_{2}=\operatorname{diag}\left(k_{21}, k_{22}, k_{23}\right)$, with $k_{1 i}$ and $k_{2 i}$, are positive gains. Differentiating (23) and substituting the result and (24) into (18), the closed-loop dynamics are obtained as

$$
\dot{s}=-K_{1} \operatorname{sign}(s)^{(p-1) / p}-K_{2} \int_{0}^{t} \operatorname{sign}^{(p-2) / p}(s(\tau)) d \tau+\widetilde{d} .
$$

Let us define $z=\left[\begin{array}{ll}z_{1 i} & z_{2 i}\end{array}\right]^{T}$ with

$$
\begin{gathered}
z_{1 i}=s_{i}, \\
z_{2 i}=-k_{2 i} \int_{0}^{t} \operatorname{sign}^{(p-2) / p}\left(s_{i}(\tau)\right) d \tau+\tilde{d}_{i}, \\
\dot{\tilde{d}}_{i}(t)=\delta_{i}(t) ;
\end{gathered}
$$

then (26) can be written in scalar form $(i=1,2,3)$ as

$$
\begin{aligned}
& \dot{z}_{1 i}=-k_{1 i}\left|z_{1 i}\right|^{(p-1) / p} \operatorname{sign}\left(z_{1 i}\right)+z_{2 i}, \\
& \dot{z}_{2 i}=-k_{2 i}\left|z_{1 i}\right|^{(p-2) / p} \operatorname{sign}\left(z_{1 i}\right)+\delta_{i} .
\end{aligned}
$$

Note that (28) is in the form of the smooth super twisting algorithm [27]. Next, the proof of finite-time convergence to the origin is given.

Theorem 4. Assume that the first-time derivative of disturbance $\widetilde{d}_{i}$ is bounded and $\left|\delta_{i}(t)\right| \leq L$, where $L$ is a positive constant. With $k_{1 i}>0, k_{2 i}>0(i=1,2,3)$, all trajectories of the system (28) converge in finite time to the region

$$
\varepsilon=\left\{\|\vartheta\| \leq\left(\frac{L\|\widetilde{q}\|}{\sigma_{\min }(\widetilde{Q})}\right)^{(p-1) /(p-2)}\right\}
$$

where $\vartheta=\left[\left|z_{1 i}\right|^{(p-1) / p} \operatorname{sign}\left(z_{1 i}\right) z_{2 i}\right]$ and $\|\vartheta\|$ denotes the Euclidean norm of $9 . \sigma_{\min }(\widetilde{Q})$ denote the minimum singular value of the matrix $\widetilde{Q}$ given by

$$
\begin{gathered}
\widetilde{Q}=\frac{k_{1 i}}{2}\left[\begin{array}{cc}
2 k_{2 i}+k_{1 i}^{2}\left(\frac{2 p-2}{p}\right) & -k_{1 i}\left(\frac{2 p-2}{p}\right) \\
-k_{1 i}\left(\frac{2 p-2}{p}\right) & \left(\frac{2 p-2}{p}\right)
\end{array}\right], \\
\widetilde{q}=\left[\begin{array}{c}
2 \\
-k_{1 i}
\end{array}\right] .
\end{gathered}
$$

Proof. We first construct the Lyapunov function by extending the ideas of Moreno and Osorio [35]. Let the Lyapunov function be chosen as

$$
\begin{aligned}
V(z)= & \frac{k_{2 i} p}{p-1}\left|z_{1 i}\right|^{2(p-1) / p}+\frac{1}{2} z_{2 i}^{2} \\
& +\frac{1}{2}\left(k_{1 i}\left|z_{1 i}\right|^{(p-1) / p} \operatorname{sign}\left(z_{1 i}\right)-z_{2 i}\right)^{2},
\end{aligned}
$$

which can be written as

$$
V(z)=\vartheta^{T} P \vartheta
$$

where $\vartheta=\left[\left|z_{1 i}\right|^{(p-1) / p} \operatorname{sign}\left(z_{1 i}\right) z_{2 i}\right]$ and

$$
P=\frac{1}{2}\left[\begin{array}{cc}
\frac{2 k_{2 i} p}{p-1}+k_{1 i}^{2} & -k_{1 i} \\
-k_{1 i} & 2
\end{array}\right] \text {. }
$$


Note that $V(z)$ is continuous but is not differentiable at $z_{1 i}=$ 0 . It is positive definite and radially unbounded if $k_{2 i}>0$; that is,

$$
\sigma_{\min }(P)\|\vartheta\|^{2} \leq V \leq \sigma_{\max }(P)\|\vartheta\|^{2},
$$

where $\|\vartheta\|^{2}=\left|z_{1 i}\right|^{2(p-1) / p}+z_{2 i}^{2} \cdot \sigma_{\text {min }}(P)$ and $\sigma_{\text {max }}(P)$ denote the minimum and maximum singular values of the matrix $P$. The derivative of $V$ can be written as

$$
\begin{aligned}
\dot{V}= & \left(\frac{k_{2 i} p}{p-1}+\frac{1}{2} k_{1 i}^{2}\right)\left(\frac{2 p-2}{p}\right)\left|z_{1 i}\right|^{(p-2) / p} \\
& \times \operatorname{sign}\left(z_{1 i}\right) \dot{z}_{1 i}+2 z_{2 i} \dot{z}_{2 i} \\
& -k_{1 i}\left|z_{1 i}\right|^{(p-1) / p} \operatorname{sign}\left(z_{1 i}\right) \dot{z}_{2 i} \\
& -k_{1 i} \frac{p-1}{p} z_{2 i}\left|z_{1 i}\right|^{-1 / p} \dot{z}_{1 i} .
\end{aligned}
$$

Substituting (28) into (32), we obtain

$$
\begin{aligned}
\dot{V}= & \left(\frac{k_{2 i} p}{p-1}+\frac{1}{2} k_{1 i}^{2}\right)\left(\frac{2 p-2}{p}\right)\left|z_{1 i}\right|^{(p-2) / p} \\
& \times \operatorname{sign}\left(z_{1 i}\right)\left(z_{2 i}-k_{1 i}\left|z_{1 i}\right|^{(p-1) / p} \operatorname{sign}\left(z_{1 i}\right)\right) \\
& +2 z_{2 i}\left(-k_{2 i}\left|z_{1 i}\right|^{(p-2) / p} \operatorname{sign}\left(z_{1 i}\right)+\delta_{i}\right) \\
& -k_{1 i}\left|z_{1 i}\right|^{(p-1) / p} \\
& \times \operatorname{sign}\left(z_{1 i}\right)\left(-k_{2 i}\left|z_{1 i}\right|^{(p-2) / p} \operatorname{sign}\left(z_{1 i}\right)+\delta_{i}\right) \\
& -k_{1 i} z_{2 i}\left(\frac{p-1}{p}\right)\left|z_{1 i}\right|^{-1 / p} \\
& \times\left(z_{2 i}-k_{2 i}\left|z_{1 i}\right|^{(p-1) / p} \operatorname{sign}\left(z_{1 i}\right)\right) \\
\dot{V}= & -\left|z_{1 i}\right|^{-1 / p}\left(-k_{1 i}^{2}\left(\frac{2 p-2}{p}\right)\right)\left|z_{1 i}\right|^{(p-1) / p} \\
& \times k_{1 i}\left|z_{1 i}\right|^{(p-1) / p} \operatorname{sign}\left(z_{1 i}\right) \delta_{i} . \\
& \times \frac{p-1}{p} k_{1 i}^{3}\left|z_{1 i}\right|^{2(p-1) / p}+k_{1 i}\left(\frac{p-1}{p}\right) z_{2 i}^{2}+2 z_{2 i} \delta_{i} \\
& \operatorname{sig} z_{2 i}+k_{1 i} k_{2 i}\left|z_{1 i}\right|^{2(p-1) / p} \\
& \\
&
\end{aligned}
$$

Also, $\dot{V}$ can be rearranged as

$$
\begin{aligned}
\dot{V} & \leq-\left|z_{1 i}\right|^{-1 / p} \vartheta^{T} \widetilde{Q} \vartheta+L \widetilde{q}^{T} \vartheta \\
& =-\left|z_{1 i}\right|^{-1 / p} \sigma_{\min }(\widetilde{Q})\|\vartheta\|^{2}+L \widetilde{q}^{T} \vartheta .
\end{aligned}
$$

From (34), it follows by $\left|z_{1 i}\right|^{-1 / p} \geq\|9\|^{-1 /(p-1)}$ and (37) that

$$
\begin{aligned}
\dot{V} & \leq-\|\vartheta\|^{-1 /(p-1)} \sigma_{\min }(\widetilde{Q})\|\vartheta\|^{2}+L\|\widetilde{q}\|\|\vartheta\| \\
& \leq-\sigma_{\min }(\widetilde{Q})\|\vartheta\|^{(2 p-3) /(p-1)}+L\|\widetilde{q}\|\|\vartheta\| \\
& \leq-\left(\sigma_{\min }(\widetilde{Q})\|\vartheta\|^{(p-2) /(p-1)}-L\|\widetilde{q}\|\right)\|\vartheta\| .
\end{aligned}
$$

Using (34), we know that $V / \sigma_{\max }(P) \leq\|\vartheta\|^{2} \leq V / \sigma_{\min }(P)$. We obtain

$$
\dot{V} \leq-\left(\sigma_{\min }(\widetilde{Q})\|\vartheta\|^{(p-2) /(p-1)}-L\|\widetilde{q}\|\right) \frac{V}{\sigma_{\max }(P)} .
$$

If $\sigma_{\min }(\widetilde{Q})\|9\|^{(p-2) /(p-1)}>L\|\widetilde{q}\|$, the error system (28) will finite-time converge to the region

$$
\varepsilon=\left\{\|\vartheta\| \leq\left(\frac{L\|\widetilde{q}\|}{\sigma_{\min }(\widetilde{Q})}\right)^{(p-1) /(p-2)}\right\}
$$

In fact, we can choose $k_{i 1}$ and $k_{2 i}$ such that $L\|\widetilde{q}\| / \sigma_{\min }(\widetilde{Q})<1$. With $p>2,(p-1) /(p-2)$ is sufficiently large; $\|\vartheta\|$ is sufficiently small in finite time.

\section{Smooth MST Controller Design}

Next, we study the modified version of the ST algorithm. To improve the dynamical performance and reduce chattering phenomenon, a controller is developed by adding linear correction terms to the nonlinear one.

The proposed controller is given by the following:

$$
\begin{aligned}
u_{f}=\left(B_{0}\right)^{-1} & \\
& \times\left(-F-\Lambda_{1} \operatorname{sign}(s)^{(p-1) / p}-\Lambda_{2} s\right. \\
& \left.\quad-\Lambda_{3} \int_{0}^{t} \operatorname{sign}(s(\tau))^{(p-2) / p} d \tau-\Lambda_{4} \int_{0}^{t} s(\tau) d \tau\right) .
\end{aligned}
$$

The gain matrices $\Lambda_{1}=\operatorname{diag}\left(\lambda_{11}, \lambda_{12}, \lambda_{13}\right), \Lambda_{2}=$ $\operatorname{diag}\left(\lambda_{21}, \lambda_{22}, \lambda_{23}\right), \Lambda_{3}=\operatorname{diag}\left(\lambda_{31}, \lambda_{32}, \lambda_{33}\right)$, and $\Lambda_{4}=$ $\operatorname{diag}\left(\lambda_{41}, \lambda_{42}, \lambda_{43}\right)$ with $\lambda_{1 i}, \lambda_{2 i}, \lambda_{3 i}$, and $\lambda_{4 i}$ are positive gains.

On substituting (41) into (18) the closed-loop dynamics are obtained as

$$
\begin{aligned}
\dot{s}= & -\Lambda_{1} \operatorname{sign}(s)^{(p-1) / p}-\Lambda_{2} s \\
& -\Lambda_{3} \int_{0}^{t} \operatorname{sign}(s(\tau))^{(p-2) / p} d \tau \\
& -\Lambda_{4} \int_{0}^{t} s(\tau) d \tau+\tilde{d} .
\end{aligned}
$$


Using the same procedure as in Section 3, (42) can be written as

$$
\begin{aligned}
& \dot{z}_{1 i}=-\lambda_{1 i}\left|z_{1 i}\right|^{(p-1) / p} \operatorname{sign}\left(z_{1 i}\right)+z_{2 i}-\lambda_{2 i} z_{i 1} \\
& \dot{z}_{2 i}=-\lambda_{3 i}\left|z_{1 i}\right|^{(p-2) / p} \operatorname{sign}\left(z_{1 i}\right)-\lambda_{4 i} z_{i 1}+\delta_{i} .
\end{aligned}
$$

The finite-time stability of the closed-loop system (43) is ensured by using the following theorem.

Theorem 5. Suppose that $\lambda_{1 i}, \lambda_{2 i}, \lambda_{3 i}, \lambda_{4 i}>0(i=1,2,3)$ are selected such that

$$
4 \lambda_{3 i} \lambda_{4 i}>\left[\frac{2 \lambda_{3 i} p}{p-1}+\lambda_{1 i}^{2}\left(\frac{4 p-2}{p}\right)^{2}\right] \lambda_{2 i}^{2}
$$

and the first-time derivative of disturbance $\tilde{d}_{i}$ is bounded and $\left|\delta_{i}(t)\right| \leq L$, where $L$ is a positive constant. Then, all trajectories of the system (43) converge in finite time to the region

$$
\begin{gathered}
\|\xi\| \leq \Delta=\min \left\{\Delta_{1}, \Delta_{2}\right\}, \quad \Delta_{1}=\frac{L\|\Gamma\|}{2 \sigma_{\min }\left(\Omega_{2}\right)}, \\
\Delta_{2}=\left(\frac{L\|\Gamma\|}{\sigma_{\min }\left(\Omega_{1}\right)}\right)^{(2 p-3) /(2 p-2)},
\end{gathered}
$$

where $\Delta_{1}, \Delta_{2}>0$ are the results from the chosen gains

$$
\begin{gathered}
\Omega_{1}=\frac{\lambda_{1 i}}{2}\left[\begin{array}{ccc}
2 \lambda_{3 i}+\lambda_{1 i}^{2}\left(\frac{2 p-2}{p}\right) & 0 & -\lambda_{1 i}\left(\frac{2 p-2}{p}\right) \\
0 & \left(2 \lambda_{4 i}+\lambda_{2 i}^{2}\left(\frac{6 p-2}{p}\right)\right) & -\lambda_{2 i}\left(\frac{4 p-2}{p}\right) \\
-\lambda_{1 i}\left(\frac{2 p-2}{p}\right) & -\lambda_{2 i}\left(\frac{4 p-2}{p}\right) & \frac{2(p-1)}{p}
\end{array}\right], \\
\Omega_{2}=\lambda_{2 i}\left[\begin{array}{ccc}
\left(\lambda_{3 i}+\lambda_{1 i}^{2}\left(\frac{3 p-2}{2}\right)\right) & 0 & 0 \\
0 & \left(\lambda_{4 i}+\lambda_{2 i}^{2}\right) & -\lambda_{2 i} \\
0 & -\lambda_{2 i} & 1
\end{array}\right] \\
\Gamma=\left[\begin{array}{ccc}
-\lambda_{1 i}-\lambda_{2 i} & 2
\end{array}\right] .
\end{gathered}
$$

Proof. Let the Lyapunov function be chosen as

$$
\begin{aligned}
V(z)= & \frac{\lambda_{3 i} p}{p-1}\left|z_{1 i}\right|^{2(p-1) / p}+\lambda_{4 i} z_{1 i}^{2}+\frac{1}{2} z_{2 i}^{2} \\
& +\frac{1}{2}\left(\lambda_{1 i}\left|z_{1 i}\right|^{(p-1) / p} \operatorname{sign}\left(z_{1 i}\right)+\lambda_{2 i} z_{1 i}-z_{2 i}\right)^{2} .
\end{aligned}
$$

The selected Lyapunov function can be expressed as

$$
\begin{aligned}
V(z)= & 2\left(\frac{\lambda_{3 i} p}{p-1}\left|z_{1 i}\right|^{2(p-1) / p}+\frac{1}{2} \lambda_{1 i}^{2}\right)+z_{2 i}^{2} \\
& +\left(\lambda_{4 i}+\frac{1}{2} \lambda_{2 i}^{2}\right) z_{1 i}^{2}+\lambda_{1 i} \lambda_{2 i}\left|z_{1 i}\right|^{(p-1) / p} \\
& \times \operatorname{sign}\left(z_{1 i}\right) z_{1 i}-\lambda_{1 i}\left|z_{1 i}\right|^{(p-1) / p} \\
& \times \operatorname{sign}\left(z_{1 i}\right) z_{2 i}-\lambda_{2 i} z_{1 i} z_{2 i},
\end{aligned}
$$

which can be written as

$$
V(z)=\xi^{T} \Pi \xi
$$

where $\xi=\left[\left|z_{1 i}\right|^{(p-1) / p} \operatorname{sign}\left(z_{1 i}\right) \quad z_{1 i} z_{2 i}\right]^{T}$ and

$$
\Pi=\frac{1}{2}\left[\begin{array}{ccc}
\frac{2 \lambda_{3 i} p}{p-1}+\lambda_{1 i}^{2} & \lambda_{1 i} \lambda_{2 i} & -\lambda_{1 i} \\
\lambda_{1 i} \lambda_{2 i} & \left(2 \lambda_{4 i}+\lambda_{2 i}^{2}\right) & -\lambda_{2 i} \\
-\lambda_{1 i} & -\lambda_{2 i} & 2
\end{array}\right] .
$$

It satisfies

$$
\sigma_{\min }(\Pi)\|\xi\|^{2} \leq V \leq \sigma_{\max }(\Pi)\|\xi\|^{2},
$$

where $\|\xi\|^{2}=\left|z_{1 i}\right|^{2(p-1) / p}+z_{1 i}^{2}+z_{2 i}^{2}$.

$$
\text { Consider }
$$

$$
\begin{aligned}
\dot{V} \leq & \left(2 \lambda_{3 i}+\lambda_{1 i}^{2} \frac{p-1}{p}\right)\left|z_{1 i}\right|^{(p-2) / p} \operatorname{sign}\left(z_{1 i}\right) \dot{z}_{1 i} \\
& +2 z_{2 i} \dot{z}_{2 i}+\left(2 \lambda_{4 i}+\lambda_{2 i}^{2}\right) z_{1 i} \dot{z}_{1 i} \\
& +\left(\frac{2 p-1}{p}\right) \lambda_{1 i} \lambda_{2 i}\left|z_{1 i}\right|^{(p-1) / p} \operatorname{sign}\left(z_{1 i}\right) \dot{z}_{1 i} \\
& -\lambda_{1 i}\left|z_{1 i}\right|^{(p-1) / p} \operatorname{sign}\left(z_{1 i}\right) \dot{z}_{2 i} \\
& -\lambda_{1 i}\left(\frac{p-1}{p}\right)\left|z_{1 i}\right|^{-1 / p} z_{2 i} \dot{z}_{1 i}-\lambda_{2 i} z_{1 i} \dot{z}_{2 i} \\
& -\lambda_{2 i} z_{2 i} \dot{z}_{1 i} .
\end{aligned}
$$


Substituting $\dot{z}_{1 i}$ and $\dot{z}_{2 i}$ into (52), we obtain

$$
\begin{aligned}
\dot{V} \leq & \left(2 \lambda_{3 i}+\lambda_{1 i}^{2} \frac{p-1}{p}\right)\left|z_{1 i}\right|^{(p-2) / p} \operatorname{sign}\left(z_{1 i}\right) \\
& \times\left(z_{2 i}-\lambda_{1 i}\left|z_{1 i}\right|^{(p-1) / p} \operatorname{sign}\left(z_{1 i}\right)-\lambda_{2 i} z_{2 i}\right) \\
& +2 z_{1 i}\left(-\lambda_{3 i}\left|z_{1 i}\right|^{(p-2) / p} \operatorname{sign}\left(z_{1 i}\right)-\lambda_{4 i} z_{1 i}+\delta_{i}\right) \\
& +\left(2 \lambda_{4 i}+\lambda_{2 i}^{2}\right) z_{1 i} \\
& \times\left(z_{2 i}-\lambda_{1 i}\left|z_{1 i}\right|^{(p-1) / p} \operatorname{sign}\left(z_{1 i}\right)-\lambda_{2 i} z_{1 i}\right) \\
& +\lambda_{1 i} \lambda_{2 i}\left|z_{1 i}\right|^{(p-1) / p} \operatorname{sign}\left(z_{1 i}\right) \\
& \times\left(z_{2 i}-\lambda_{1 i}\left|z_{1 i}\right|^{(p-1) / p} \operatorname{sign}\left(z_{1 i}\right)-\lambda_{2 i} z_{1 i}\right) \\
& +\lambda_{1 i}\left|z_{1 i}\right|^{(p-1) / p} \operatorname{sign}\left(z_{1 i}\right) \\
& \times\left(-\lambda_{3 i}\left|z_{1 i}\right|^{(p-2) / p} \operatorname{sign}\left(z_{1 i}\right)-\lambda_{4 i} z_{1 i}+\delta_{i}\right) \\
& \times\left(z_{2 i}-\lambda_{1 i}\left|z_{1 i}\right|^{(p-1) / p} \operatorname{sign}\left(z_{1 i}\right)-\lambda_{2 i} z_{1 i}\right) \\
& -\lambda_{1 i} z_{2 i} \frac{p-1}{p}\left|z_{1 i}\right|^{-1 / p} z_{1 i}\left(-\lambda_{3 i}\left|z_{1 i}\right|^{(p-2) / p} \operatorname{sign}\left(z_{1 i}\right) \lambda_{4 i} z_{1 i}+\delta_{i}\right) \\
& \left.+\lambda_{1 i}\left|z_{1 i}\right|^{(p-1) / p} \operatorname{sign}\left(z_{1 i}\right)-\lambda_{2 i} z_{1 i}\right)
\end{aligned}
$$

After lengthy algebraic manipulation, one obtains

$$
\begin{aligned}
\dot{V} \leq & -\left|z_{1 i}\right|^{-1 / p} \\
\times & \left(\left[\lambda_{1 i} \lambda_{3 i}+\lambda_{1 i}^{3} \frac{(p-1)}{p}\right]\left|z_{1 i}\right|^{2(p-1) / p}\right. \\
& -2 \lambda_{1 i}^{2} \frac{(p-1)}{p}\left|z_{1 i}\right|^{(p-1) / p} \\
& \times \operatorname{sign}\left(z_{1 i}\right) z_{2 i}+\left[\lambda_{1 i} \lambda_{4 i}+\frac{1}{2} \lambda_{1 i} \lambda_{1 i}^{2} \frac{(6 p-2)}{p}\right] z_{1 i}^{2} \\
& \left.-2 \lambda_{1 i} \lambda_{2 i} \frac{(4 p-2)}{p} z_{1 i} z_{2 i}+\frac{(p-1)}{p} \lambda_{1 i} z_{2 i}^{2}\right) \\
- & \left(\left[\lambda_{2 i} \lambda_{3 i}+\lambda_{2 i}^{3} \frac{(3 p-2)}{p}\right]\left|z_{1 i}\right|^{2(p-1) / p}\right.
\end{aligned}
$$

$$
\begin{array}{r}
\left.+\left(\lambda_{2 i} \lambda_{4 i}+\lambda_{2 i}^{3}\right) z_{1 i}^{2}+2 \lambda_{2 i}^{2} z_{1 i} z_{2 i}-\lambda_{2 i} z_{2 i}^{2}\right) \\
-\delta_{i}\left|z_{1 i}\right|^{(p-1) / p} \operatorname{sign}\left(z_{1 i}\right)-\delta_{i} \lambda_{2 i} z_{1 i}+2 \delta_{i} z_{2 i},
\end{array}
$$

which can be written as

$$
\dot{V}=-\left|z_{1 i}\right|^{-1 / p} \xi^{T} \Omega_{1} \xi-\xi^{T} \Omega_{2} \xi+\delta^{T} \Gamma \xi .
$$

Note that with positive values of $\lambda_{1 i}, \lambda_{2 i}, \lambda_{3 i}$, and $\lambda_{4 i}$, it can be ensured that $\Omega_{2}$ is positive definite. Also, the condition (44) is required to guarantee that $\Omega_{1}$ is positive definite.

Therefore, we have

$$
\begin{aligned}
\dot{V} \leq & -\left|z_{1 i}\right|^{-1 / p} \sigma_{\min }\left(\Omega_{1}\right)\|\xi\|^{2}-\sigma_{\min }\left(\Omega_{2}\right)\|\xi\|^{2} \\
& +L\|\Gamma\|\|\xi\| .
\end{aligned}
$$

Using $\left|z_{1 i}\right|^{-1 / p} \geq\|\xi\|^{-1 /(p-1)}$, one obtains

$$
\begin{aligned}
\dot{V} \leq & -\left|z_{1 i}\right|^{-1 / p} \sigma_{\min }\left(\Omega_{1}\right)\|\xi\|^{2}-\sigma_{\min }\left(\Omega_{2}\right)\|\xi\|^{2} \\
& +L\|\Gamma\|\|\xi\| \\
\leq & -\sigma_{\min }\left(\Omega_{1}\right)\|\xi\|^{(2 p-3) /(p-1)}-\sigma_{\min }\left(\Omega_{2}\right)\|\xi\|^{2} \\
& +L\|\Gamma\|\|\xi\| .
\end{aligned}
$$

We can change (57) into the following forms:

$$
\begin{gathered}
\dot{V} \leq-\|\xi\|\left(\sigma_{\min }\left(\Omega_{1}\right)\|\xi\|^{(p-2) /(p-1)}-L\|\Gamma\|\right)-\sigma_{\min }\left(\Omega_{2}\right)\|\xi\|^{2} \\
\dot{V} \leq-\sigma_{\min }\left(\Omega_{1}\right)\|\xi\|^{(2 p-3) /(p-1)}-\|\xi\|\left(\sigma_{\min }\left(\Omega_{2}\right)\|\xi\|-L\|\Gamma\|\right) .
\end{gathered}
$$

Therefore, if we choose the gains such that

$$
\begin{gathered}
\sigma_{\min }\left(\Omega_{1}\right)-\frac{L\|\Gamma\|}{\|\xi\|^{(2 p-3) /(p-1)}}>0 \\
\sigma_{\min }\left(\Omega_{2}\right)-\frac{L\|\Gamma\|}{\|\xi\|}>0
\end{gathered}
$$

then the finite-time stability is guaranteed. Let positive scalars $\eta_{1}$ and $\eta_{2}$ be defined as

$$
\begin{gathered}
\eta_{1}=\sigma_{\min }\left(\Omega_{1}\right)-\frac{L\|\Gamma\|}{\|\xi\|^{(2 p-3) /(p-1)}}, \\
\eta_{2}=\sigma_{\min }\left(\Omega_{2}\right)-\frac{L\|\Gamma\|}{\|\xi\|} .
\end{gathered}
$$

Then, (58) can be respectively written as

$$
\begin{gathered}
\dot{V} \leq-\eta_{1}\|\xi\|^{(2 p-3) /(p-1)}-\sigma_{\min }\left(\Omega_{2}\right)\|\xi\|^{2} \\
\dot{V} \leq-\sigma_{\min }\left(\Omega_{1}\right)\|\xi\|^{(2 p-3) /(p-1)}-\eta_{1}\|\xi\|^{2} .
\end{gathered}
$$


From (51), we get $V / \sigma_{\max }(\Pi) \leq\|\xi\|^{2} \leq V / \sigma_{\min }(\Pi)$. Therefore, we obtain

$$
\begin{aligned}
& \dot{V} \leq-\left(\frac{\eta_{1}}{\left(\sigma_{\max }(\Pi)\right)^{(2 p-3) /(2 p-2)}}\right) V^{(2 p-3) /(2 p-2)}-\frac{\sigma_{\min }\left(\Omega_{2}\right)}{\sigma_{\max }(\Pi)} V \\
& \dot{V} \leq-\left(\frac{\sigma_{\min }\left(\Omega_{1}\right)}{\left(\sigma_{\max }(\Pi)\right)^{(2 p-3) /(2 p-2)}}\right) V^{(2 p-3) /(2 p-2)}-\frac{\eta_{2}}{\sigma_{\max }(\Pi)} V .
\end{aligned}
$$

Therefore, if we choose $\lambda_{1 i}, \lambda_{2 i}, \lambda_{3 i}$, and $\lambda_{4 i}$ such that the conditions (59) are satisfied, the finite-time convergence property can be obtained. Meanwhile, the gains guarantee the system trajectory will finite-time converge to the region

$$
\begin{gathered}
\|\xi\| \leq \Delta=\min \left\{\Delta_{1}, \Delta_{2}\right\}, \\
\Delta_{1}=\frac{L\|\Gamma\|}{2 \sigma_{\min }\left(\Omega_{2}\right)}, \quad \Delta_{2}=\left(\frac{L\|\Gamma\|}{\sigma_{\min }\left(\Omega_{1}\right)}\right)^{(2 p-3) /(2 p-2)} .
\end{gathered}
$$

In fact, we can choose $\lambda_{1 i}, \lambda_{2 i}, \lambda_{3 i}$, and $\lambda_{4 i}$ such that $(L\|\Gamma\|) /\left(\sigma_{\min }\left(\Omega_{1}\right)\right)<1$. With $p>2,(2 p-3) /(2 p-2)$ is sufficiently large, so that $\Delta_{2}$ can be greatly reduced with $\Delta_{2} \ll \Delta_{1}$. This means that the proposed control has strong robustness and disturbance rejection ability.

For (56), we can find that when the system state is far from the origin, $-\left|z_{1 i}\right|^{-1 / p} \sigma_{\min }\left(\Omega_{1}\right)\|\xi\|^{2}$ is small and $-\sigma_{\min }\left(\Omega_{2}\right)\|\xi\|^{2}$ is large, so the behavior of $-\sigma_{\min }\left(\Omega_{2}\right)\|\xi\|^{2}$ is strong. On the other hand around, the origin, $-\left|z_{1 i}\right|^{-1 / p} \sigma_{\min }\left(\Omega_{1}\right)\|\xi\|^{2}$ is large and $-\sigma_{\min }\left(\Omega_{2}\right)\|\xi\|^{2}$ is small, so the behavior of $-\left|z_{1 i}\right|^{-1 / p} \sigma_{\min }\left(\Omega_{1}\right)\|\xi\|^{2}$ is strong. Hence, the behaviors are strong during the whole convergence.

\section{Simulation Results}

An example of a rigid-body satellite [10] is presented with numerical simulations to compare the performance of the developed controllers (24) and (41) with the controller (1) in [20]. The spacecraft is assumed to have the nominal inertia matrix

$$
J=\left[\begin{array}{ccc}
20 & 1.2 & 0.9 \\
1.2 & 17 & 1.4 \\
0.9 & 1.4 & 15
\end{array}\right] \mathrm{kg} \cdot \mathrm{m}^{2}
$$

and the parameter uncertainties

$$
\Delta J=\operatorname{diag}[\sin (0.1 t) 2 \sin (0.2 t) \quad 3 \sin (0.2 t)] \mathrm{kg} \cdot \mathrm{m}^{2} .
$$

The attitude control problem is considered in the presence of external disturbance $d(t)$. The external disturbances are described as

$$
d(t)=\left[\begin{array}{l}
0.1 \sin (0.1 t) \\
0.2 \sin (0.2 t) \\
0.3 \sin (0.3 t)
\end{array}\right] \mathrm{N}-\mathrm{m} .
$$

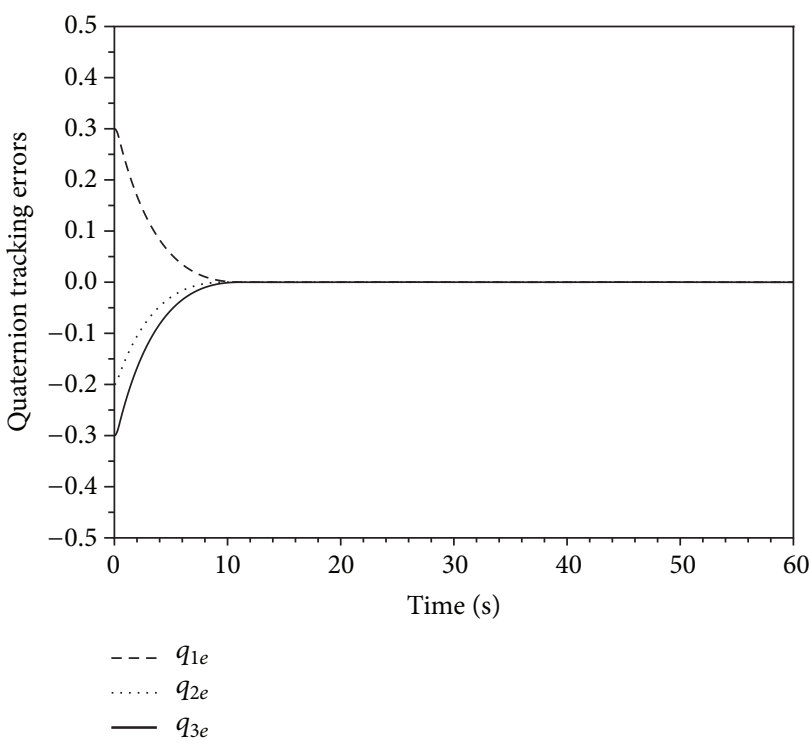

FIGURE 1: Quaternion tracking errors-Controller (1) in [20].

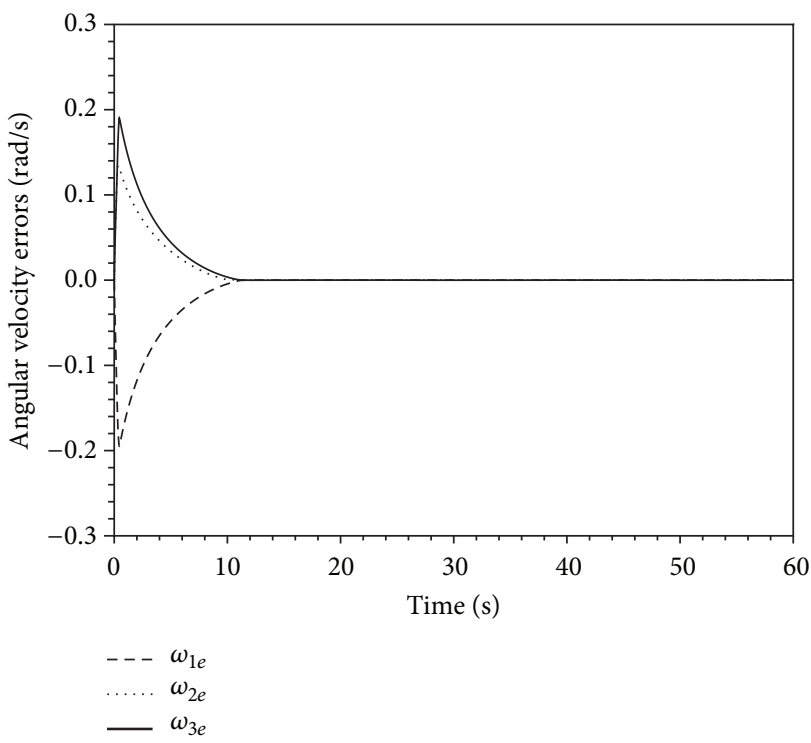

FIGURE 2: Angular velocity tracking errors-Controller (1) in [20].

In this numerical simulation, we assume that the desired angular velocity is given by

$$
\omega_{r}(t)=0.05\left[\begin{array}{c}
\sin \left(\frac{\pi t}{100}\right) \\
\sin \left(\frac{2 \pi t}{100}\right) \\
\sin \left(\frac{3 \pi t}{100}\right)
\end{array}\right] \mathrm{rad} / \mathrm{s} .
$$

The initial condition of the unit quaternion is $Q(0)=[0.3-$ $\left.\begin{array}{lll}0.2 & -0.3 & 0.8832\end{array}\right]^{T}$ and the initial target unit quaternion is $Q_{r}(0)=\left[\begin{array}{llll}0 & 0 & 0 & 1\end{array}\right]^{T}$. The initial value of the angular velocity is $\omega(0)=\left[\begin{array}{lll}0 & 0 & 0\end{array}\right]^{T} \mathrm{rad} / \mathrm{s}$. 


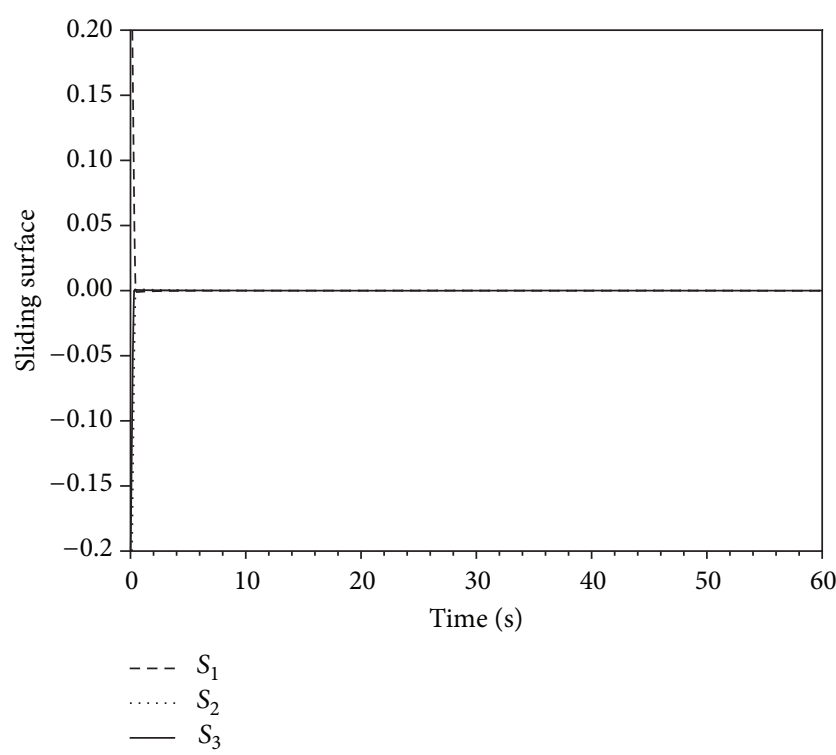

FIGURE 3: Switching function-Controller (1) in [20].

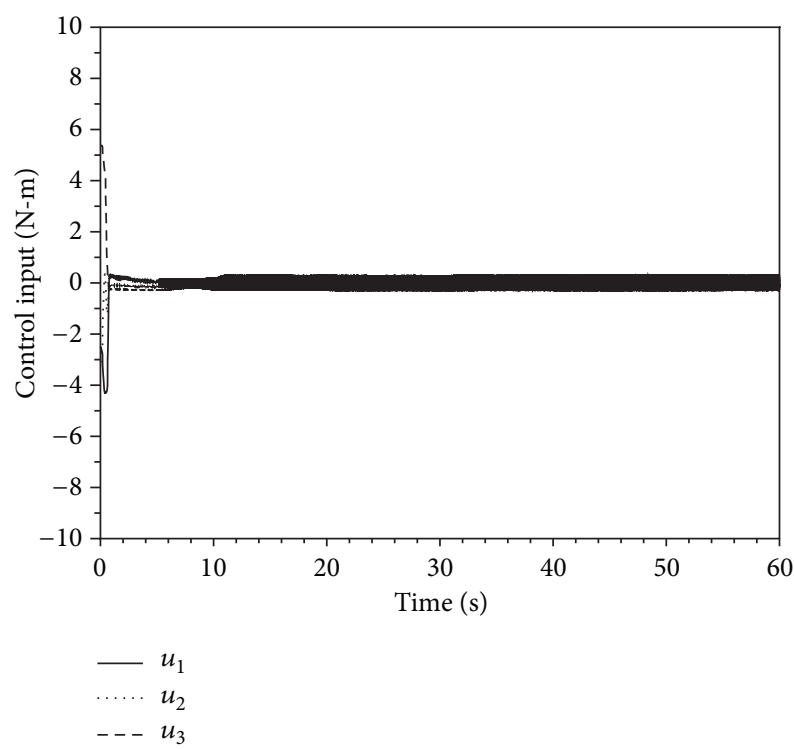

FIgURE 4: Control torques-Controller (1) in [20].

For the smooth ST controller (24), the chosen gains are given as $K_{1}=\operatorname{diag}(2.0,2.0,2.0)$ and $K_{2}=\operatorname{diag}(2.5,2.5,2.5)$. On the other hand, for the smooth MST controller (41), the control gains are selected to be $\Lambda_{1}=\operatorname{diag}(2.0,2.0,2.0)$, $\Lambda_{2}=\operatorname{diag}(0.5,0.5,0.5), \Lambda_{3}=\operatorname{diag}(2.0,2.0,2.0)$, and $\Lambda_{4}=$ $\operatorname{diag}(1.5,1.5,1.5)$. For both control algorithms, we use the same sliding manifold (23). The corresponding parameters in controller (1) in [20] are selected.

Simulation results with the controller (1) in [20] are presented in Figures 1-4. As shown in Figures 1 and 2, attitude and angular velocity tracking errors converge to zero in about 15 seconds. Figure 3 shows very the fast convergence property of the controller (1) in [20]. From Figure 4, it can be seen that chattering cannot be avoided. The boundary layer

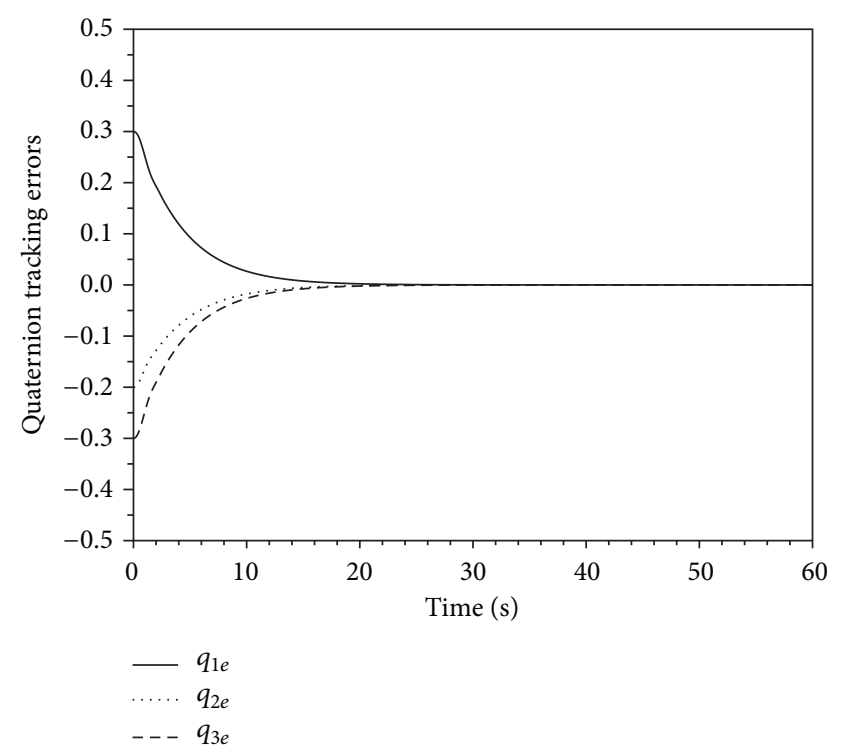

FIGURE 5: Quaternion tracking errors — smooth ST controller (24).

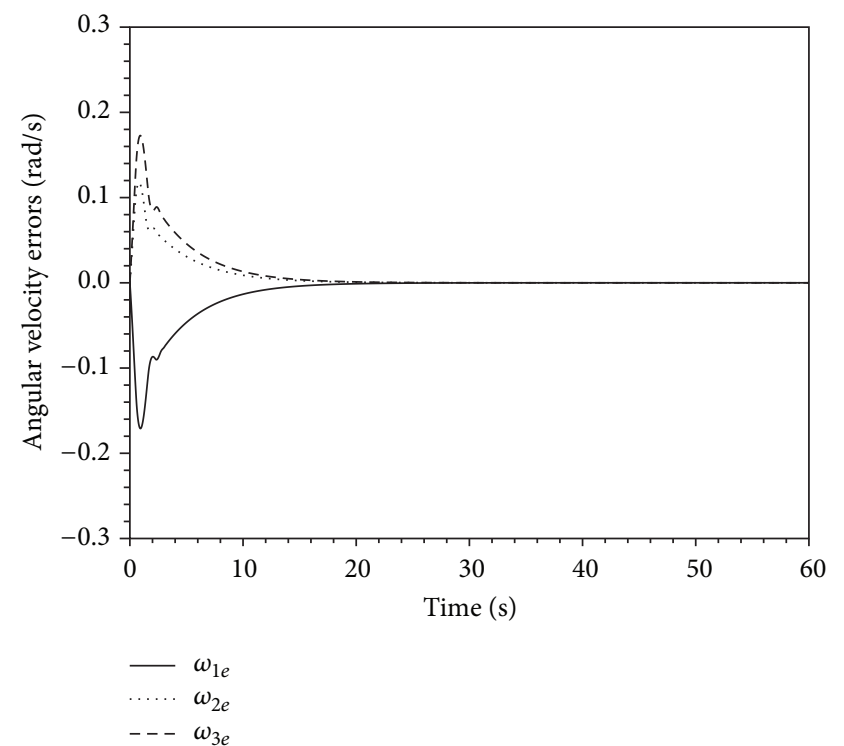

FIGURE 6: Angular velocity tracking errors-smooth ST controller (24).

$\|s\| \leq 1.9 \times 10^{-4}$ is reached in finite time. Regarding the accuracy, the bounds on $\left\|q_{e}\right\|$ and $\left\|\omega_{e}\right\|$ are $\left\|q_{e}\right\| \leq 7.1 \times 10^{-5}$ and $\left\|\omega_{e}\right\| \leq 1.1 \times 10^{-4}$ with the sampling time $h=0.005$.

For simulation results with the controllers (24) and (41), the attitude quaternion tracking errors are shown in Figures 5 and 9, and angular velocity tracking errors are illustrated in Figures 6 and 10. The smooth MST controller (41) gives smoother angular velocity tracking outputs than the controller in (24). From Figures 7 and 11, it can be seen that the sliding vectors are on the sliding surface $s=0$ after 5 seconds. As shown in Figures 8 and 12, the response of the control variables obtained by the controller (24) has a quick change in a short time for the first 5 seconds, while the controller 


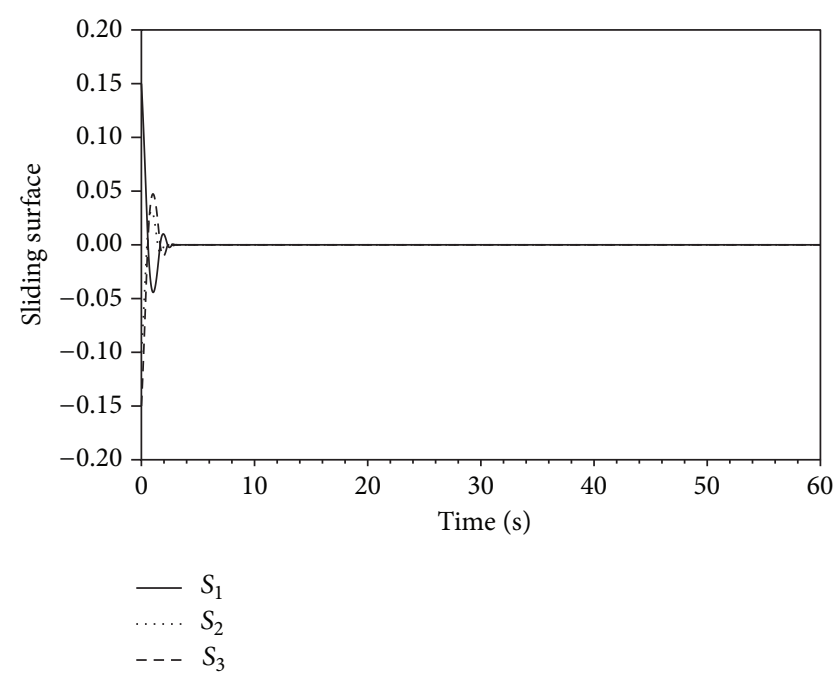

FIGURE 7: Switching function-smooth ST controller (24).

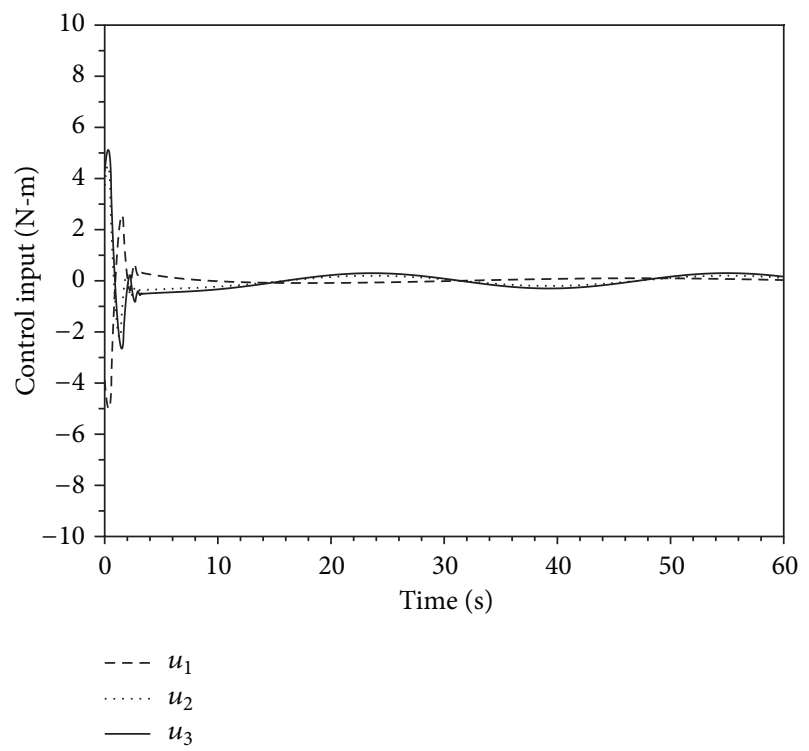

FIGURE 8: Control torques-smooth ST controller (24).

(41) provides smoother responses of control torques. For the controller (24), the boundary layer $\|s\| \leq 5 \times 10^{-7}$ is achieved in finite time. Regarding the accuracy, the bounds on $\left\|q_{e}\right\|$ and $\left\|\omega_{e}\right\|$ are $\left\|q_{e}\right\| \leq 2 \times 10^{-7}$ and $\left\|\omega_{e}\right\| \leq 6 \times 10^{-7}$ with the sampling time $h=0.005$. Also, for the controller (41), the boundary layer $\|s\| \leq 3.2 \times 10^{-7}$ is reached in finite time. Regarding the accuracy, the bounds on $\left\|q_{e}\right\|$ and $\left\|\omega_{e}\right\|$ are $\left\|q_{e}\right\| \leq 9.9 \times 10^{-8}$ and $\left\|\omega_{e}\right\| \leq 2 \times 10^{-7}$ with the sampling time $h=0.005$.

A comparison of the simulation results obtained by the smooth ST controller, smooth MST controller, and the controller (1) in [20] shows the following. Although the controller (1) in [20] gives the fastest convergence rate, it can be seen that the undesired chattering is not avoided.

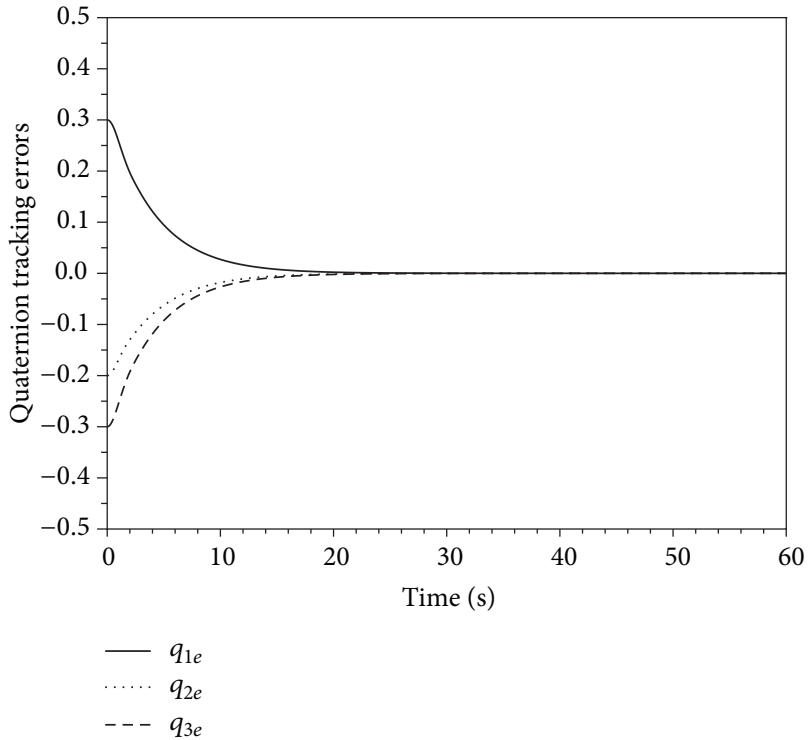

FIGURE 9: Quaternion tracking errors-smooth MST controller (41).

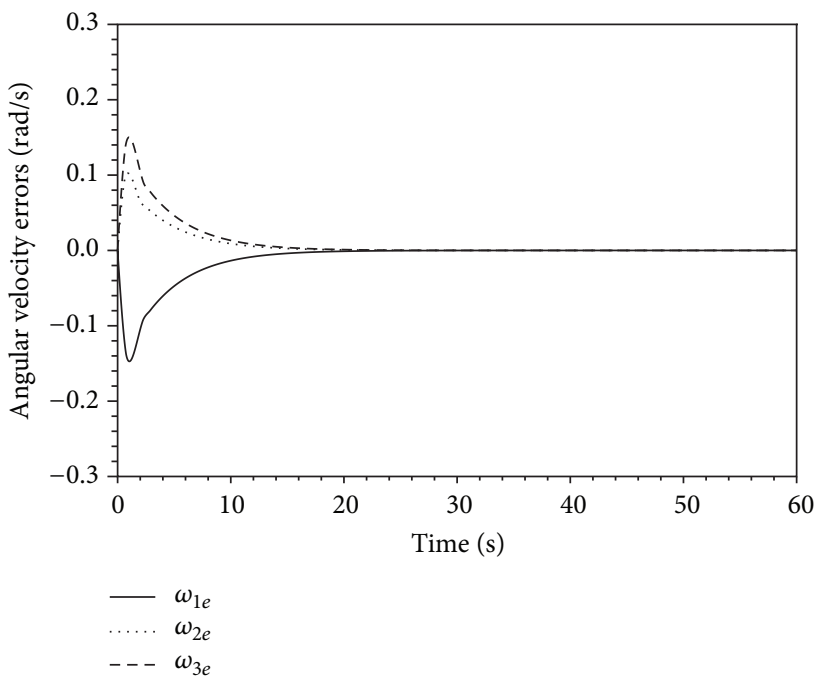

Figure 10: Angular velocity tracking errors-smooth MST controller (41).

Moreover, it cannot provide high-precision tracking performance. The smooth MST controller gives outstanding accuracy and provides smoother response of control torques when compared with the smooth ST approach. In view of these simulation results, the smooth MST controller (41) seems to give the best overall control for practical attitude tracking control of a rigid spacecraft.

\section{Conclusion}

The proposed finite robust attitude tracking controllers have been successfully applied to spacecraft tracking maneuvers. For the first controller, the smooth ST control is applied to deal with quaternion-based spacecraft-attitude-tracking 


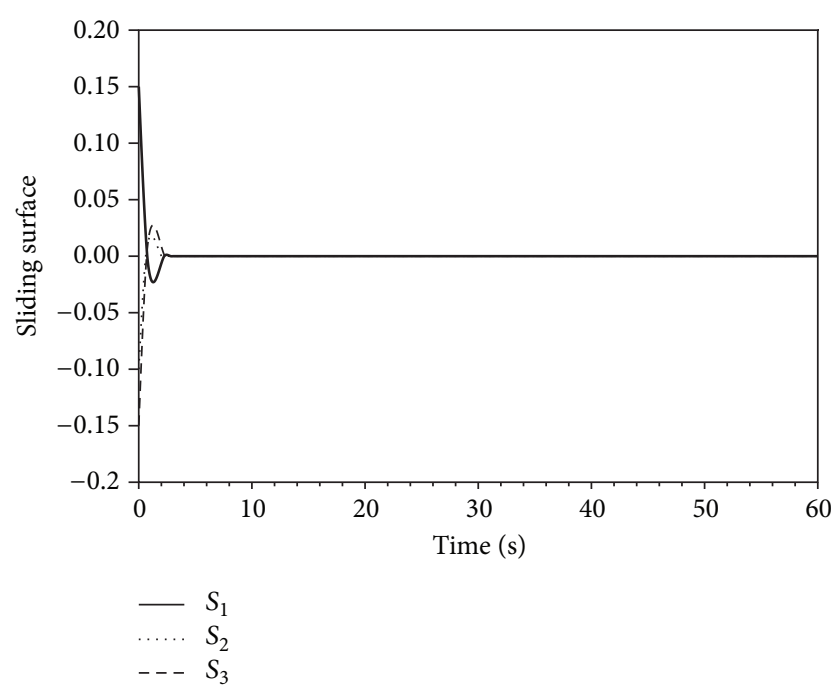

FIGURE 11: Switching function-smooth MST controller (41).

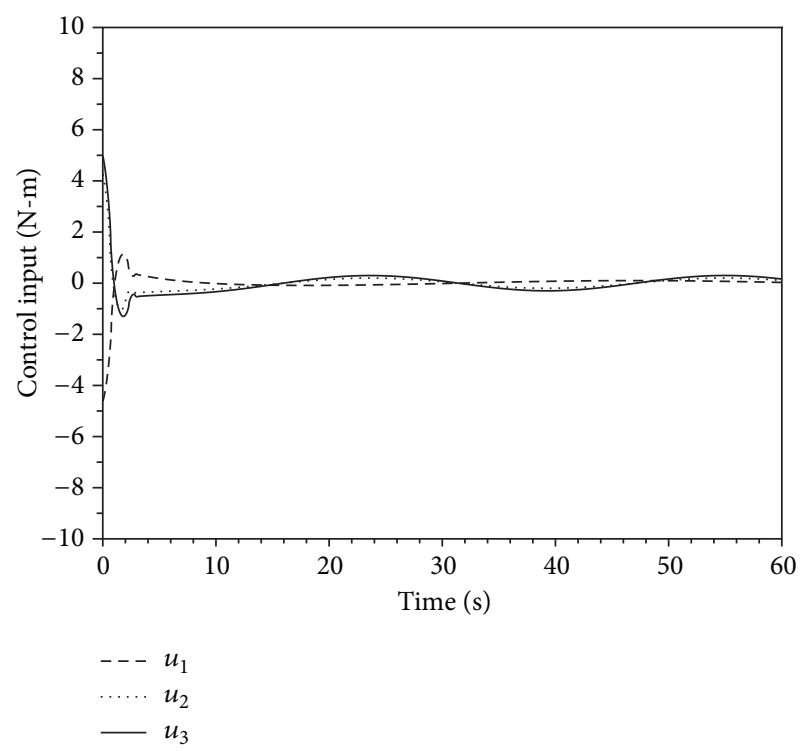

FIgURE 12: Control torques-smooth MST controller (41).

maneuvers. The second controller is developed by adding the linear correction terms to the first super twisting control algorithm to improve the dynamic performance of the closedloop system. The concepts of the strong Lyapunov function are employed to ensure a finite-time property of the proposed controllers. The presented methods provide very good accuracy of tracking results and reduce the undesired chattering. Moreover, strong robustness ability is obtained and is shown by using proposed Lyapunov functions. Numerical simulations are also given to demonstrate the performance of the proposed control laws.

\section{References}

[1] J. S.-C. Yuan, "Closed-loop manipulator control using quaternion feedback," IEEE Journal of Robotics and Automation, vol. 4, no. 4, pp. 434-440, 1988.
[2] S. M. Joshi, A. G. Kelkar, and J. T.-Y. Wen, "Robust attitude stabilization of spacecraft using nonlinear quaternion feedback," IEEE Transactions on Automatic Control, vol. 40, no. 10, pp. 1800-1803, 1995.

[3] W. Cai, X. H. Liao, and Y. D. Song, "Indirect robust adaptive fault-tolerant control for attitude tracking of spacecraft," Journal of Guidance, Control, and Dynamics, vol. 31, no. 5, pp. 1456-1463, 2008.

[4] Y. D. Song and W. Cai, "Quaternion observer-based modelindependent attitude tracking control of spacecraft," Journal of Guidance, Control, and Dynamics, vol. 32, no. 5, pp. 1476-1482, 2009.

[5] J. Su and K.-Y. Cai, "Globally stabilizing proportional-integralderivative control laws for rigid-body attitude tracking," Journal of Guidance, Control, and Dynamics, vol. 34, no. 4, pp. 1260$1264,2011$.

[6] J. T.-Y. Wen and K. Kreutz-Delgado, "The attitude control problem," IEEE Transactions on Automatic Control, vol. 36, no. 10, pp. 1148-1162, 1991.

[7] P. Pisu and A. Serrani, "Attitude tracking with adaptive rejection of rate gyro disturbances," IEEE Transactions on Automatic Control, vol. 52, no. 12, pp. 2374-2379, 2007.

[8] L.-L. Show, J.-C. Juang, and Y.-W. Jan, "An LMI-based nonlinear attitude control approach," IEEE Transactions on Control Systems Technology, vol. 11, no. 1, pp. 73-83, 2003.

[9] W. Luo, Y.-C. Chu, and K.-V. Ling, "Inverse optimal adaptive control for attitude tracking of spacecraft," IEEE Transactions on Automatic Control, vol. 50, no. 11, pp. 1639-1654, 2005.

[10] Z. Chen and J. Huang, "Attitude tracking and disturbance rejection of rigid spacecraft by adaptive control," IEEE Transactions on Automatic Control, vol. 54, no. 3, pp. 600-605, 2009.

[11] H. Wong, M. S. de Queiroz, and V. Kapila, "Adaptive tracking control using synthesized velocity from attitude measurements," Automatica, vol. 37, no. 6, pp. 947-953, 2001.

[12] B. T. Costic, D. M. Dawson, M. S. de Queiroz, and V. Kapila, "A quaternion-based adaptive attitude tracking controller without velocity measurements," in Proceedings of the 39th IEEE Confernce on Decision and Control, pp. 2424-2429, Sydney, Australia, December 2000.

[13] I. Ali, G. Radice, and J. Kim, "Backstepping control design with actuator torque bound for spacecraft attitude maneuver," Journal of Guidance, Control, and Dynamics, vol. 33, no. 1, pp. 254-259, 2010.

[14] J. D. Bošković, S.-M. Li, and R. K. Mehra, "Robust tracking control design for spacecraft under control input saturation," Journal of Guidance, Control, and Dynamics, vol. 27, no. 4, pp. 627-633, 2004.

[15] F.-K. Yeh, "Sliding-mode adaptive attitude controller design for spacecrafts with thrusters," IET Control Theory and Applications, vol. 4, no. 7, pp. 1254-1264, 2010.

[16] Z. H. Man, A. P. Paplinski, and H. R. Wu, "A robust MIMO terminal sliding mode control scheme for rigid robotic manipulators," IEEE Transactions on Automatic Control, vol. 39, no. 12, pp. 2464-2469, 1994.

[17] Y. Feng, X. Yu, and Z. Man, "Non-singular terminal sliding mode control of rigid manipulators," Automatica, vol. 38, no. 12, pp. 2159-2167, 2002.

[18] E. Jin and Z. Sun, "Robust controllers design with finite time convergence for rigid spacecraft attitude tracking control," Aerospace Science and Technology, vol. 12, no. 4, pp. 324-330, 2008 . 
[19] Y. Xia, Z. Zhu, M. Fu, and S. Wang, "Attitude tracking of rigid spacecraft with bounded disturbances," IEEE Transactions on Industrial Electronics, vol. 58, no. 2, pp. 647-659, 2011.

[20] S. Li, Z. Wang, and S. Fei, "Comments on the paper: robust controllers design with finite time convergence for rigid spacecraft attitude tracking control," Aerospace Science and Technology, vol. 15, no. 3, pp. 193-195, 2011.

[21] W. Perruquetti and J. P. Barbot, Sliding Mode Control in Engineering, Marcel Dekker, New York, NY, USA, 2002.

[22] C. Edwards, E. Fossas Colet, and L. Fridman, Advances in Variable Structure and Sliding Mode Control, Lecture Notes in Control and Information Sciences, Springer, Berlin, Germany, 2006.

[23] A. Damiano, G. L. Gatto, I. Marongiu, and A. Pisano, "Secondorder sliding-mode control of dc drives," IEEE Transactions on Industrial Electronics, vol. 51, no. 2, pp. 364-373, 2004.

[24] G. Bartolini, A. Pisano, and E. Usai, "Second-order slidingmode control of container cranes," Automatica, vol. 38, no. 10, pp. 1783-1790, 2002.

[25] M. Canale, L. Fagiano, A. Ferrara, and C. Vecchio, "Vehicle yaw control via second-order sliding-mode technique," IEEE Transactions on Industrial Electronics, vol. 55, no. 11, pp. 39083916, 2008.

[26] C. Pukdeboon, A. S. I. Zinober, and M.-W. L. Thein, "Quasicontinuous higher order sliding-mode controllers for spacecraft-attitude-tracking maneuvers," IEEE Transactions on Industrial Electronics, vol. 57, no. 4, pp. 1436-1444, 2010.

[27] Y. B. Shtessel, I. A. Shkolnikov, and A. Levant, "Smooth secondorder sliding modes: Missile guidance application," Automatica, vol. 43, no. 8, pp. 1470-1476, 2007.

[28] Y. Orlov, "Finite time stability and robust control synthesis of uncertain switched systems," SIAM Journal on Control and Optimization, vol. 43, no. 4, pp. 1253-1271, 2005.

[29] A. Polyakov and A. Poznyak, "Lyapunov function design for finite-time convergence analysis: "twisting" controller for second-order sliding mode realization," Automatica, vol. 45, no. 2, pp. 444-448, 2009.

[30] V. T. Haimo, "Finite time controllers," SIAM Journal on Control and Optimization, vol. 24, no. 4, pp. 760-770, 1986.

[31] J. R. Wertz, Spacecraft Attitude Determination and Control, Kluwer Academic Publishers, 1978.

[32] M. J. Sidi, Spacecraft Dynamics and Control, Cambridge University Press, Cambridge, Mass, USA, 1997.

[33] M. D. Shuster, "A survey of attitude representations," Journal of the Astronautical Sciences, vol. 41, no. 4, pp. 439-517, 1993.

[34] S. P. Bhat and D. S. Bernstein, "Finite-time stability of continuous autonomous systems," SIAM Journal on Control and Optimization, vol. 38, no. 3, pp. 751-766, 2000.

[35] J. A. Moreno and M. Osorio, "A Lyapunov approach to secondorder sliding mode controllers and observers," in Proceedings of the 47th IEEE Conference on Decision and Control (CDC '08), pp. 2856-2861, Cancun, Mexico, December 2008. 


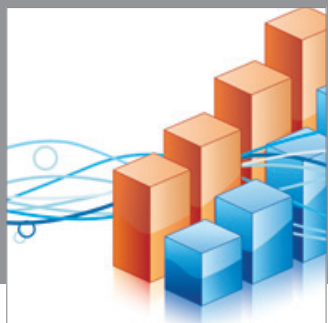

Advances in

Operations Research

mansans

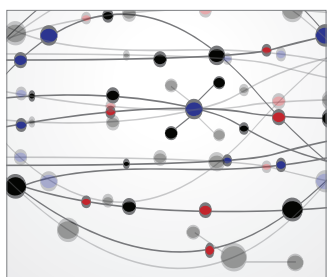

The Scientific World Journal
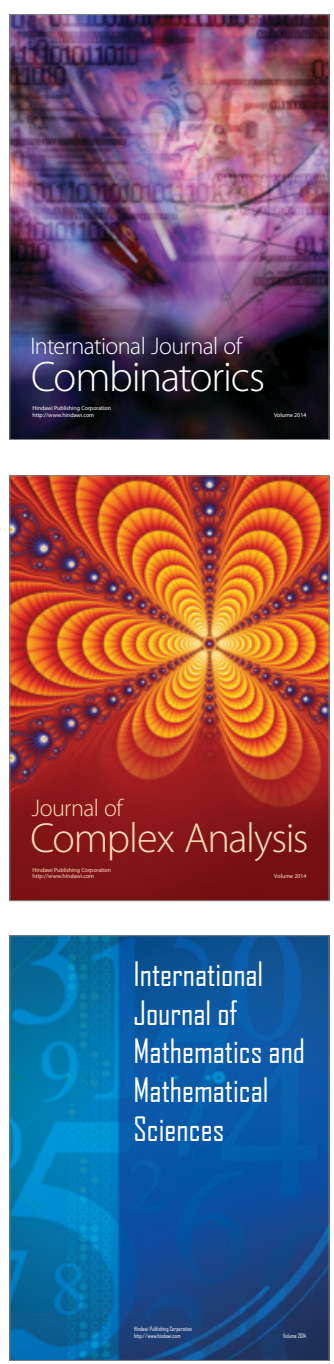
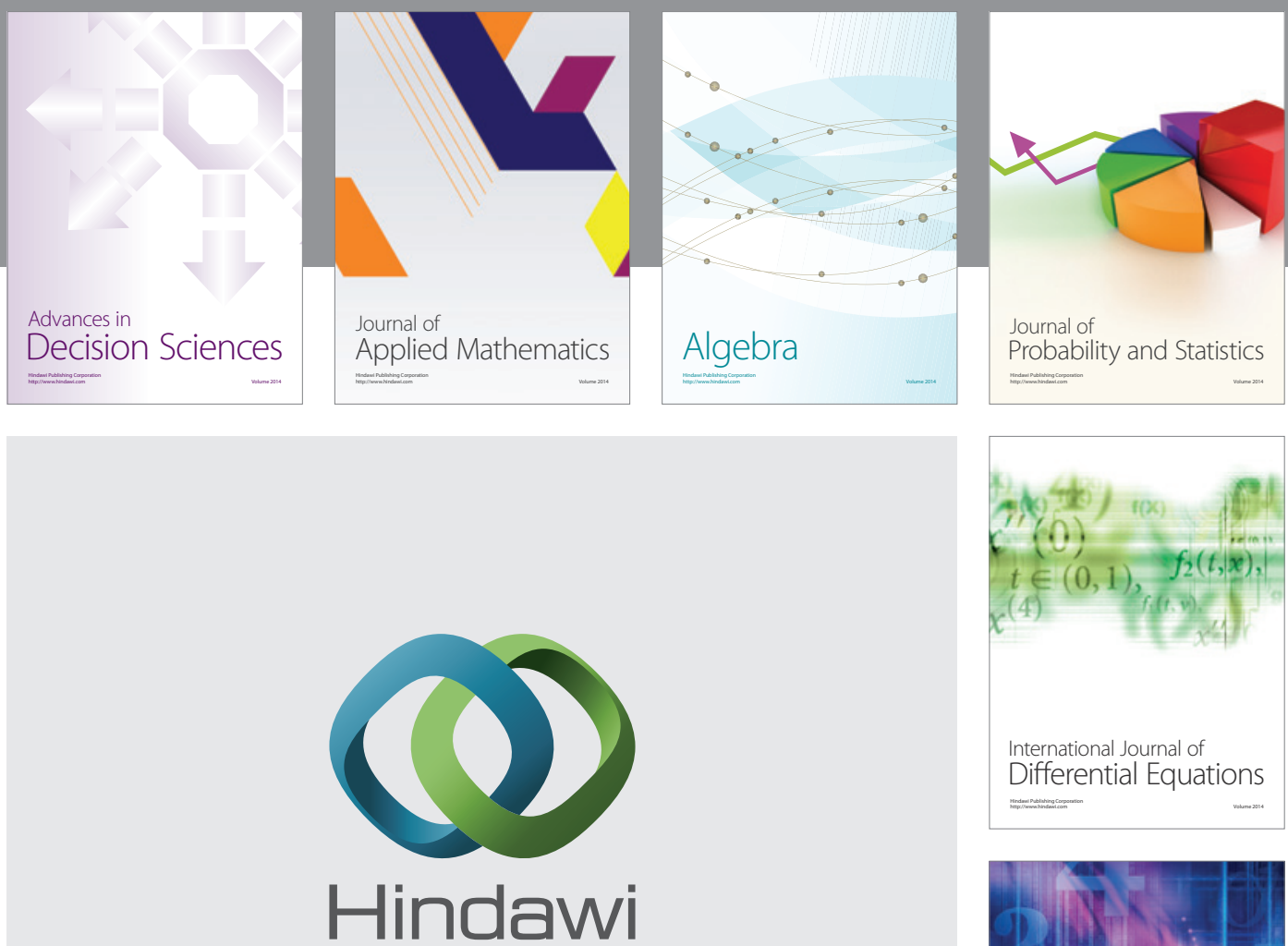

Submit your manuscripts at http://www.hindawi.com
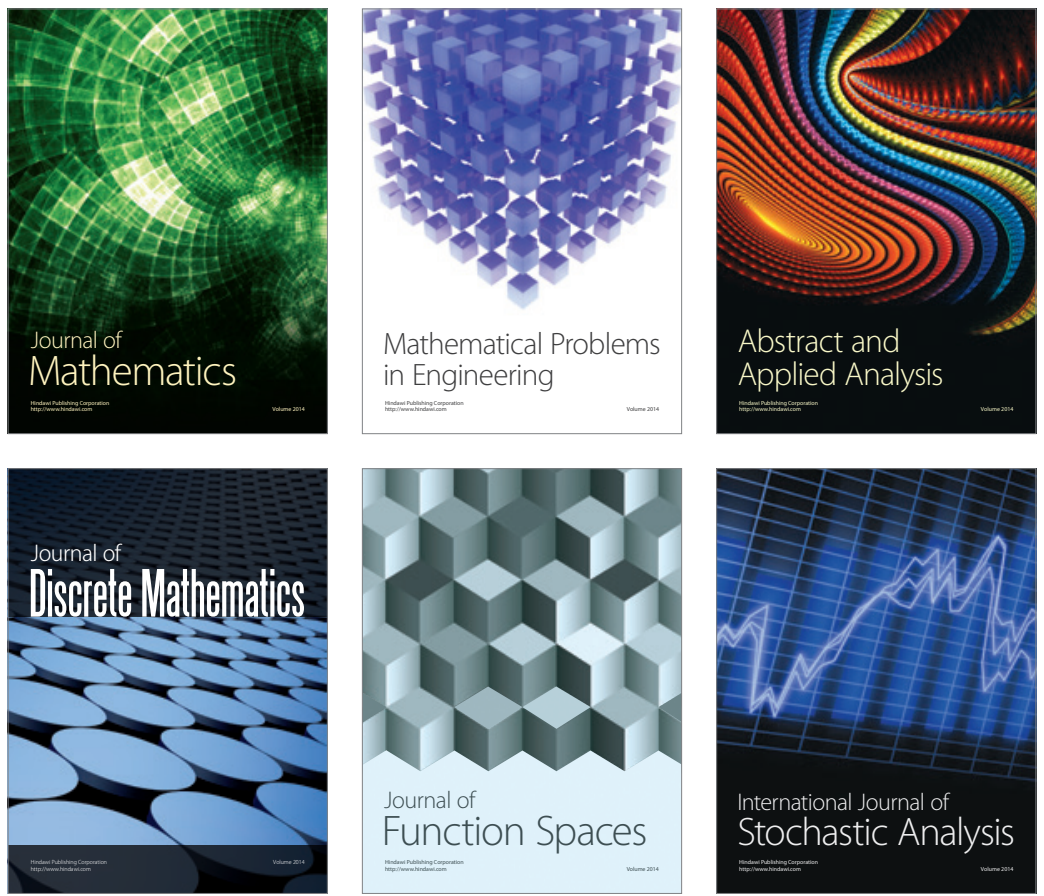

Journal of

Function Spaces

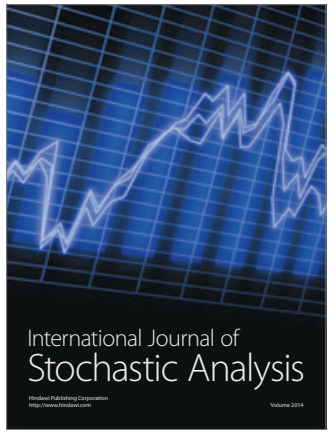

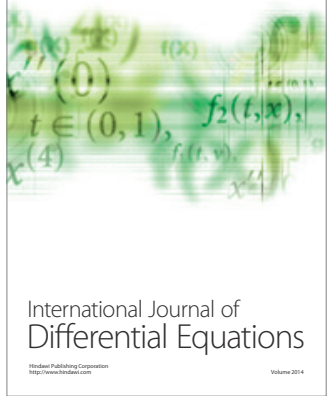
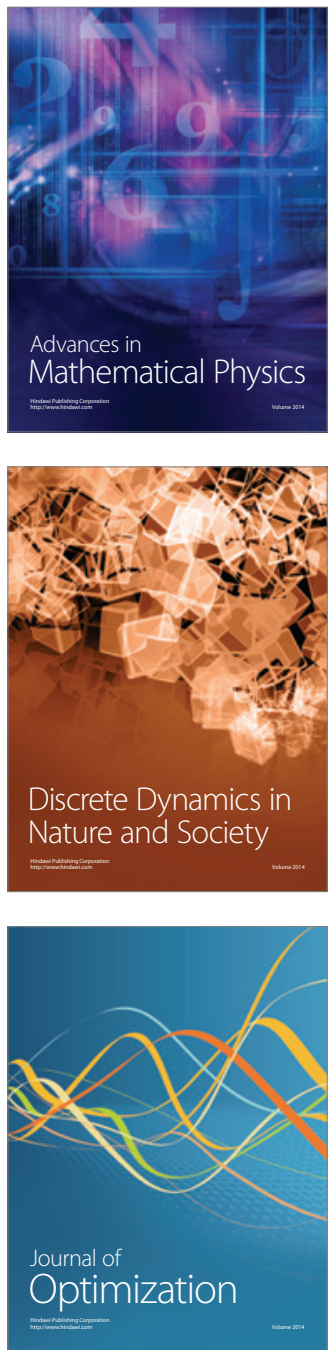